\title{
A narrative review of Barrett's esophagus in 2020, molecular and clinical update
}

\author{
Aamir N. Dam, Jason Klapman \\ Department of Gastrointestinal Oncology, Moffitt Cancer Center, Tampa, FL, USA \\ Contributions: (I) Conception and design: All authors; (II) Administrative support: None; (III) Provision of study materials or patients: None; (IV) \\ Collection and assembly of data: All authors; (V) Data analysis and interpretation: All authors; (VI) Manuscript writing: All authors; (VII) Final \\ approval of manuscript: All authors. \\ Correspondence to: Jason Klapman, MD, FASGE, AGAF. Medical Director in the Department of Gastrointestinal Oncology, Moffitt Cancer Center, \\ Professor of Medicine, Department of Oncologic Sciences at the University of South Florida, 12902 Magnolia Drive, Tampa, FL 33612, USA. \\ Email: jason.klapman@moffitt.org.
}

\begin{abstract}
Barrett's esophagus (BE) is a condition resulting from an acquired metaplastic epithelial change in the esophagus in response to gastroesophageal reflux. BE is the only known precursor lesion to esophageal adenocarcinoma, and can progress from non-dysplastic BE (NDBE) to low grade dysplasia (LGD) and high grade dysplasia (HGD), and ultimately invasive carcinoma. Although the risk of developing esophageal adenocarcinoma (EAC) in NBDE is less than $0.5 \%$ per year, there has been a rising incidence of EAC in Western countries, which continue to drive efforts to optimize screening and surveillance methods. The current gold standard for diagnosis is esophagogastroduodenoscopy (EGD), and there has been significant interest in alternative, minimally invasive methods for screening which would be more readily accessible in the primary care setting. Surveillance endoscopy in 3-5 years is recommended for NDBE given the low progression to EAC. The mainstay of treatment for LGD and HGD is endoscopic eradication therapy (EET). Visible lesions are treated with endoscopic mucosal resection (EMR) or endoscopic submucosal dissection (ESD). Radiofrequency ablation (RFA) is considered first line therapy for flat dysplastic BE and cryotherapy has shown promising results as an alternate form of treatment for of dysplasia. The molecular progression of $\mathrm{BE}$ to $\mathrm{EAC}$ is a complex process involving multiple pathways involving genetic and epigenetic modifications. Genomic studies have further led to the understanding of the complex molecular landscape that occurs early and late in the disease process. Promising biomarker panels have been investigated to help with the diagnosis of $\mathrm{BE}$ as well as aid in the risk stratification of BE during surveillance. In addition, clinical prediction models have been developed to categorize BE patients in low, intermediate, and high risk for progression to HGD and EAC. Further clinical and translational research is needed to help refine markers and techniques in diagnosis, screening, and surveillance.
\end{abstract}

Keywords: Barrett's esophagus (BE); low grade dysplasia (LGD); high grade dysplasia (HGD); endoscopic eradication therapy; esophageal cancer; biomarkers

Submitted Jun 01, 2020. Accepted for publication Jul 24, 2020.

doi: $10.21037 / \mathrm{atm}-20-4406$

View this article at: http://dx.doi.org/10.21037/atm-20-4406

\section{Introduction}

Barrett's esophagus (BE) is a condition resulting from chronic esophageal injury causing a change in the lining of the esophagus from normal squamous epithelium to a metaplastic columnar epithelium (1). The prevalence of $\mathrm{BE}$ is estimated to be $1.6 \%$ in the general population, and higher in patients with gastroesophageal reflux disease (GERD) (2-4). Risk factors associated with development of BE include increasing age, chronic GERD, male gender, 
tobacco usage, Caucasian race, family history of BE, and central obesity $(1,5)$. BE is the only recognized precursor lesion that can progress to esophageal adenocarcinoma (EAC). While the risk of nondysplastic BE developing EAC is less than $0.5 \%$ per year, the increasing incidence of EAC and dismal 5 -year overall survival rate of $<20 \%$, support the need for improved screening and surveillance methods. In addition to histologic and anatomic considerations for $\mathrm{BE}$ and EAC, a deeper understanding of the disease process at a molecular level will inherently lead to improve strategies for early detection and prevention.

In this review article, we will discuss the diagnosis, screening, natural history, clinical management and molecular features of $\mathrm{BE}$. We present the following article in accordance with the Narrative Review reporting checklist (available at http://dx.doi.org/10.21037/atm-20-4406).

\section{Methods}

We conducted a literature search for papers published up to April 2020 on the clinical and molecular aspects of BE using PubMed. The following medical subject heading terms were included for a MEDLINE search: "Barrett's esophagus", "Barrett's esophagus/diagnosis", "Barrett's esophagus/ screening", "Barrett's esophagus/molecular", "Barrett's esophagus/prevalence", "Barrett's esophagus/management", "Barrett's esophagus/biomarkers", "Barrett's esophagus/ dysplasia", "Barrett's esophagus/radiofrequency ablation", "Barrett's esophagus/cryotherapy", "Barrett's esophagus/ endoscopic mucosal resection", "Barrett's esophagus/p53", "Barrett's esophagus/microRNA", "Barrett's esophagus/ DNA methylation", "esophageal adenocarcinoma". Qualitative and quantitative data was extracted through interpretation of each article.

\section{Discussion}

\section{Diagnosis}

According to the American College of Gastroenterology (ACG), the diagnosis of BE is established if the squamocolumnar junction is displaced $\geq 1 \mathrm{~cm}$ proximal to the gastroesophageal junction (GEJ) characterized by columnar lined mucosa with histologic confirmation of intestinal metaplasia (IM) containing goblet cells (1). In the US, experts agree the diagnosis of $\mathrm{BE}$ requires histologic confirmation of IM (with goblet cells) as it confers a higher risk for cancer compared to columnar mucosa without goblet cells, although this idea has been debated in the literature $(6,7)$. The detection of IM is increased with the number of biopsies obtained (8). In patients with suspected $\mathrm{BE}$, at least 8 random biopsies should be obtained for BE segment length $<2 \mathrm{~cm}$, and 4 samples obtained every $2 \mathrm{~cm}$ in those suspected with long segment BE. Societal guidelines recommend against routine biopsy of a normal or mildly irregular Z-line because IM of the gastric cardia is common in chronic GERD patients (1).

$\mathrm{BE}$ is classified as short and long-segment based upon the length of salmon-colored mucosa measured proximal to the GEJ. Long segment BE is defined as any segment $\geq 3 \mathrm{~cm}$ and short segment is defined as $<3 \mathrm{~cm}$ (Figure 1). Standardizing reporting of BE using the Prague classification system is recommended. The Prague classification describes the circumferential and maximal extent of BE using endoscopic landmarks including the diaphragmatic pinch, GEJ and the squamocolumnar junction (9). Documenting the extent of BE is important as increasing length of $\mathrm{BE}$ has been shown to be associated with an increased risk of dysplasia or EAC (10).

\section{Screening}

Screening for BE is recommended for men with chronic and/or frequent GERD symptoms and two or more risk factors for BE (1). Screening in women is not routinely recommended and can be considered in individual cases when multiple risk factors are present. There is a significant interest in the role of screening for $\mathrm{BE}$ as the incidence of EAC has been on the rise in the US during the last four decades (11). In addition, a significant proportion of patients who develop EAC, do not have a prior diagnosis of $\mathrm{BE}$ and are not in a surveillance program (12). The goal of screening is for detection of esophageal cancer at an earlier stage with options of curative therapy.

The ideal screening test must be safe, effective, tolerable, cost effective and readily available (13). At this time, the recommended screening test is an esophagogastroduodenoscopy (EGD) with biopsies of visible columnar mucosa in the esophagus (1). Endoscopy is generally a safe procedure, although expensive and usually requires the use of sedation. The adverse events during upper endoscopy are small although include bleeding, infection, perforation and cardiopulmonary complications related to sedation (14). Despite EGD being the gold standard for screening, $25 \%$ of EACs are diagnosed within 1 year after the index endoscopy and dysplasia/EAC is 

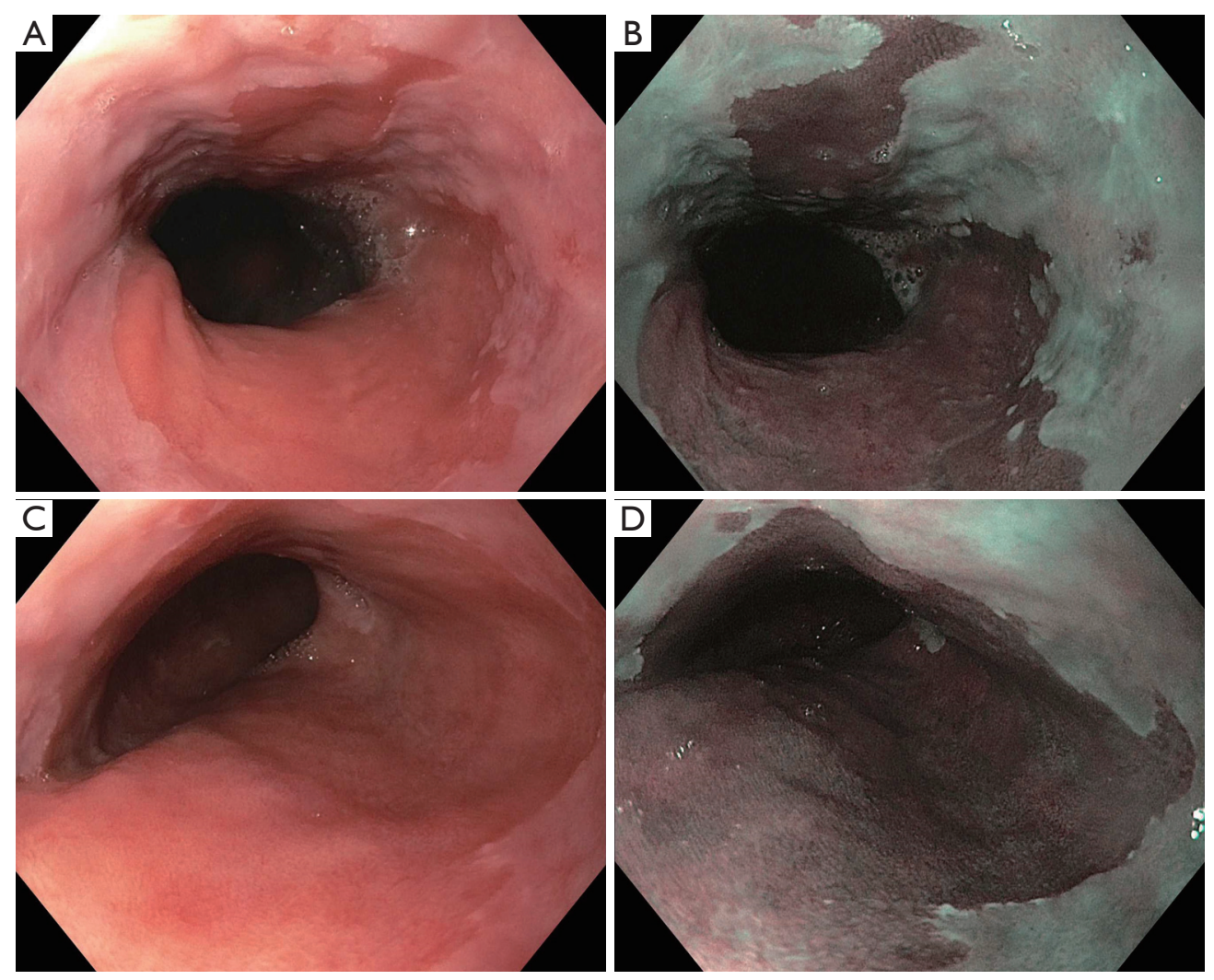

Figure 1 Classification of Barrett's esophagus. (A) High definition white light endoscopy (HDWLE) view of a short segment Barrett's esophagus (BE); (B) Narrow-band imaging (NBI) view of a short segment BE segment; (C) HDWLE view of a long segment BE; (D) NBI of a long segment BE.

commonly missed at initial BE diagnosis $(15,16)$. These studies highlight the limitations with EGD and that it is operator dependent with the chance of sampling error if careful inspection has not been performed.

Several alternative endoscopic screening methods have been explored (Table 1). The only alternative method recommended by ACG guidelines is transnasal endoscopy although has not gained widespread use in clinical practice at this time. Other promising methods include a disposable transnasal endoscopy, and video capsule endomicroscopy, although further studies are needed to validate these technologies $(17,18)$.

Non-endoscopic technologies have also gained significant interest as they are minimally invasive and have the potential for widespread applicability in the primary care setting. The most studied device is the cytosponge which is an encapsulated sponge tethered to a string. The sponge is swallowed and withdrawn out of the esophagus and collects cells from the esophageal lumen (20). It has been combined with a molecular biomarker, Trefoil Factor 3 (TFF3), and found to be accurate for diagnosing BE with a sensitivity of $80 \%$ detecting BE of any length, $87 \%$ for circumferential BE $>3 \mathrm{~cm}$, and specificity of $92 \%$ (19). Currently a multi-site randomized control trial is underway to evaluate the ability of the Cytospone-TFF3 test to identify BE among patients with GERD symptoms in the primary care setting (21). Other non-endoscopic cell collection devices have been combined with methylated DNA markers and microRNA (miRNA) markers and have shown promising results (22-25). In addition, a proof of concept study demonstrated the ability to detect $\mathrm{BE}$ by measuring unique volatile organic compounds in exhaled air samples using an electronic nose device with an accuracy of $81 \%$ (26).

\section{Natural bistory of BE}

$\mathrm{BE}$ is the only known precursor lesion to EAC and is thought to occur through a sequential progression from 
columnar intestinal metaplasia to dysplasia to invasive carcinoma. Multiple risk factors have been associated with development of BE $(1,5,27)$ (Table 2). There is a positive relationship between the number of risk factors and the prevalence of BE (5).

Non-dysplastic BE (NDBE) sequentially progresses to LGD and then to HGD before developing into the invasive cancer. Rates of progression of NDBE to EAC are approximately $0.2-0.5 \%$ per year. The rate of progression of LGD to HGD/EAC have varied between $0.4 \%$ and $13.4 \%$. The higher rate of progression in LGD was found

Table 1 Current and emerging screening techniques for Barrett's esophagus

\begin{tabular}{ll}
\hline Endoscopic methods (17,18) & \multicolumn{1}{c}{$\begin{array}{c}\text { Non-endoscopic methods } \\
(17-19)\end{array}$} \\
\hline $\begin{array}{l}\text { High definition while light } \\
\text { endoscopy }\end{array}$ & $\begin{array}{l}\text { Cytosponge and Trefoil } \\
\text { factor-3 (TFF3) }\end{array}$ \\
Transnasal Endoscope & EsophaCap \\
Video capsule endoscopy & EsoCheck balloon \\
Video capsule & Electronic nose (exhaled \\
endomicroscopy & VOCs) \\
& Methylated DNA markers \\
& MicroRNAs \\
\hline
\end{tabular}

VOCs, volatile organic compounds. in whom the diagnosis of LGD was confirmed after expert histological review and in patients with persistent LGD on subsequent endoscopies (33-36). The rates of progression from HGD to EAC is approximately $5-8 \%$ per year $(37,38)$. In a recent study, Parasa et al. developed a scoring system (Progression in BE score) based on factors including male sex, history of smoking, length of BE segment, and baseline LGD that identified patients with $\mathrm{BE}$ at low, intermediate, and high risk for HGD or EAC (28).

\section{Surveillance}

The aim of surveillance in BE is the early detection of dysplasia. A recent systematic review by Codipilly et al. showed that BE surveillance was associated with a reduction in EAC-related mortality, although the results were susceptible to lead-time and length time bias and there was substantial heterogeneity among the studies (39). Despite limitations in the evidence, societal guidelines still recommend surveillance in patients with NDBE, given that improvement in EAC survival has been observed with early staged disease and a prior diagnosis of $\mathrm{BE}(40,41)$.

Dysplasia is classified as NDBE, indeterminate, LGD, HGD, or carcinoma. The degree of dysplasia should be confirmed by an expert GI pathologist given high rate of interobserver variability (42). In a large community-based cohort of BE, LGD was over diagnosed and downgraded

Table 2 Risk factors associated with BE

\begin{tabular}{lccc}
\hline Risk factor & Risk factor for BE (1,5) & $\begin{array}{c}\text { Risk factor progression of BE to } \\
\text { HGD/EAC }(27,28)\end{array}$ & $\begin{array}{c}\text { Risk factor for recurrence of BE } \\
\text { after EET (29-32) }\end{array}$ \\
\hline $\begin{array}{l}\text { Male sex } \\
\text { Increasing age }\end{array}$ & + & + \\
GERD & + & + \\
Cigarette smoking & + & + \\
Central obesity & + & \\
Family history of BE & + & + \\
White race & + & + \\
Hiatal hernia size & & + \\
Confirmed/persistent LGD \\
HGD/MC \\
Longer BE segment length
\end{tabular}

BE, Barrett's esophagus; GERD, gastroesophageal reflux disease; EET, endoscopic eradication therapy; EAC, esophageal adenocarcinoma; HGD, high grade dysplasia; IMC, intramucosal adenocarcinoma; LGD, low grade dysplasia. 
to a diagnosis of no dysplasia in $85 \%$ of cases (34). Further studies also revealed that the majority of patients with a diagnosis of LGD in the community will be downstaged after expert review (33).

Endoscopic surveillance should utilize high-resolution/ high-definition white light endoscopy (HDWLE) for visualization of mucosa. The examination requires careful inspection of the full segment of $\mathrm{BE}$ and incorporates a retroflexed view of the GEJ. A critical step during endoscopic inspection is to identify any visible lesions or mucosal abnormalities as they have a higher tendency for harboring malignancy. Any mucosal abnormalities that are detected should undergo endoscopic mucosal resection (EMR) for diagnostic, prognostic and therapeutic purposes (1). Peters et al. evaluated 150 EMR specimens of BE neoplasia, and showed histologic evaluation led to a change in diagnosis in $49 \%$ of focal lesions and change in treatment plan in $30 \%$ of cases (43).

If no visible abnormalities are detected, four-quadrant random biopsies every $2 \mathrm{~cm}$ in NDBE and every $1 \mathrm{~cm}$ in patients with a history of dysplastic BE is recommended based on the Seattle biopsy protocol (1). More recently the role of wide-area transepithelial sampling with computerassisted analysis (WATS-3D) used adjunctively to random biopsies has been investigated. The technique involves brush cytology sampling and 3D computer-assisted analysis with the idea of improving sampling from a larger surface area. In a recent multicenter prospective trial involving community endoscopists, utilizing WATS as an adjunct, improved detection of both dysplasia and $\mathrm{BE}$ in patients undergoing screening and surveillance (44). The American Society for Gastrointestinal Endoscopy (ASGE) has made a conditional recommendation for the use of WATS-3D at this time, as an adjunct to current surveillance strategies (45).

Advanced endoscopic imaging techniques in conjunction with HDWLE have been evaluated to improved detection of dysplasia. A meta-analysis showed advanced imaging techniques (chromoendoscopy or electronic chromoendoscopy) significantly increase the diagnostic yield of dysplasia or cancer with BE by $34 \%$ (46). Narrow band imaging (NBI) display is the most widely used form of virtual chromoendoscopy that works by filtering white light into specific wavelengths and enhances the mucosal pit pattern and superficial vasculature of the mucosa (47). A wide variety of other image enhancement techniques have been studied including dye based chromoendoscopy, autofluorescence endoscopy, confocal laser endomicroscopy (CLE) and volumetric laser endomicroscopy. The ASGE developed the Preservation and Incorporation of Valuable endoscopic Innovations (PIVI) initiative designed to establish diagnostic and/or therapeutic thresholds for endoscopic technologies to be considered appropriate for clinical practice. Currently, acetic acid chromoendoscopy, NBI, and CLE are the only imaging technologies endorsed by the ASGE as they have met the required PIVI thresholds (48). Recent ASGE guidelines recommended using chromoendoscopy or virtual chromoendoscopy in conjunction with HDWLE and biopsies according to Seattle protocol in patients with $\mathrm{BE}$ undergoing surveillance for dysplasia (45).

Surveillance intervals are determined by degree of dysplasia. Current societal guidelines recommend endoscopic surveillance at 3-5 years intervals in patients without dysplasia $(1,45)$. For patients found to be indefinite for dysplasia, optimization of antisecretory therapy with a proton pump inhibitor (PPI) to reduce any inflammation from reflux disease and repeat endoscopy in 3-6 months is favored. If confirmed as indefinite for dysplasia, a surveillance interval at 12 months is recommended (1). For confirmed LGD or HGD, endoscopic eradication therapy is the preferred treatment modality, although surveillance is an acceptable option for patients with LGD $(1,37)$.

\section{Therapy}

\section{Chemoprevention}

Currently, societal guidelines recommend all patients with $\mathrm{BE}$ should receive once-daily PPI therapy. Increasing dose to twice-daily therapy is only recommended for poor control of reflux symptoms or esophagitis (1).

\section{Endoscopic eradication therapy}

Endoscopic eradication therapy (EET) is the standard management strategy for BE with dysplasia (LGD or HGD) and intramucosal cancer (IMC) (Table 3). Management begins with careful evaluation of the BE segment. Any mucosal abnormalities including nodularity, raised or irregular appearing mucosa should undergo EMR to treat BE neoplasia $(1,37,50)$. For larger lesions not amenable to conventional EMR or lesions with surface abnormalities to suggest submucosal involvement, endoscopic submucosal dissection (ESD) can also be considered at an expert center and can provide an en-bloc specimen for analysis (51). Endoscopic resection offers both therapeutic benefit and prognostic information after histologic evaluation. In patients with early EAC found on pathology, the depth of 
Table 3 Summary of Management of BE

\begin{tabular}{ll}
\hline Histologic grade & Management strategy $(1,37,49)$ \\
\hline NDBE & Surveillance endoscopy every 3-5 years \\
Indefinite for dysplasia & Optimized acid suppression therapy and repeat EGD in 3-6 months. \\
LGD & Confirm diagnosis by expert gastrointestinal pathologist.
\end{tabular}

Repeat examination under optimal acid suppression within 3-6 months with HDWLE and preferably optical chromoendoscopy. EMR or ESD of any visible lesions. Endoscopic ablation therapy of residual flat BE segment with the goal of CE-D and CEIM on subsequent sessions.

Alternatively, surveillance is an acceptable option every 6 months for the first year, then annually thereafter. If persistent LGD on repeat surveillance endoscopy, the benefits and risks of EET and ongoing surveillance should be discussed.

HGD

Confirm diagnosis by expert gastrointestinal pathologist.

Repeat examination within 6-8 weeks with HDWLE and preferably optical chromoendoscopy. EMR or ESD of any visible lesions.

Endoscopic ablation therapy of residual flat BE segment with the goal of CE-D and CEIM on subsequent sessions.

T1a esophageal cancer EMR or ESD of the visible lesion to confirm staging. If T1a confirmed on pathology with negative margins, (IMC) ablation of residual flat BE segment with the goal of CE-D and CEIM on subsequent sessions

T1b esophageal cancer

EMR or ESD of the visible lesion can be considered. If $\mathrm{T} 1 \mathrm{~b}$ confirmed and favorable pathologic features (negative margins, submucosal invasion $<500 \mu \mathrm{m}$ [sm1], well or moderately differentiated, absent lymphovascular invasion), can consider EET on case-by-case basis after multidisciplinary tumor board discussion.

If T1bsm2-3 (deeper submucosal invasion) or poor pathologic features, referral to surgical oncology for esophagectomy.

BE, Barrett's esophagus; CE-D, complete eradication of dysplasia; CEIM, complete eradication of intestinal metaplasia; EET, endoscopic eradication therapy; EMR, endoscopic mucosal resection; ESD, endoscopic submucosal dissection (ESD); EGD, esophagogastroduodenoscopy; HDWLE, high definition white light endoscopy; HGD, high grade dysplasia; IMC, intramucosal adenocarcinoma; LGD, low grade dysplasia; NBDE, non-dysplastic Barrett's esophagus; sm, submucosal

invasion determines the overall $\mathrm{T}$ staging and next step in management. Lesions confined to the mucosa with negative deep margins (T1a) are considered to be curative and have a low risk of lymph node involvement $(<2 \%)$. After successful endoscopic resection, the remaining flat BE segment should be treated with ablation therapy to achieve complete eradication of IM $(1,37,50)$ (Figure 2). Lesions with superficial submucosal invasion (T1b sm1) have conflicting results regarding rate of lymph node invasion and the decision regarding management is determined after a multidisciplinary discussion with surgical oncology. Favorable pathologic features include low grade of the lesion (well-moderate differentiation), sm1 infiltration $(<500 \mu \mathrm{m}$ invasion in the submucosa), absent lymphovascular invasion, and negative tumor margins. If these features are present, endoscopic management may be considered as an alternative strategy to esophagectomy in select patients at higher surgical risk. Lesions with deep submucosal invasion (T1b sm2 or T1b sm3) have high rates of lymphatic involvement and endoscopic therapy is inadequate $(1,37)$ (Figure 3 ).

The goal of treatment of non-nodular dysplastic $\mathrm{BE}$ is to eradicate dysplasia to prevent progression to invasive cancer. Multiple modalities have been studied in the literature and include radiofrequency ablation (RFA), cryotherapy, photodynamic therapy, argon plasma coagulation (APC), and hybrid APC, although there is a lack of head-to-head data comparing their effectiveness. Currently, societal guidelines recommend RFA as the first line treatment for dysplastic BE due to the large body of level 1 evidence on this technique $(1,37)$. During EET, optimal acid-suppressant therapy is necessary for healing and neosquamous regeneration and twice daily PPI is recommended (37).

RFA is an endoscopic technique that involves thermal 

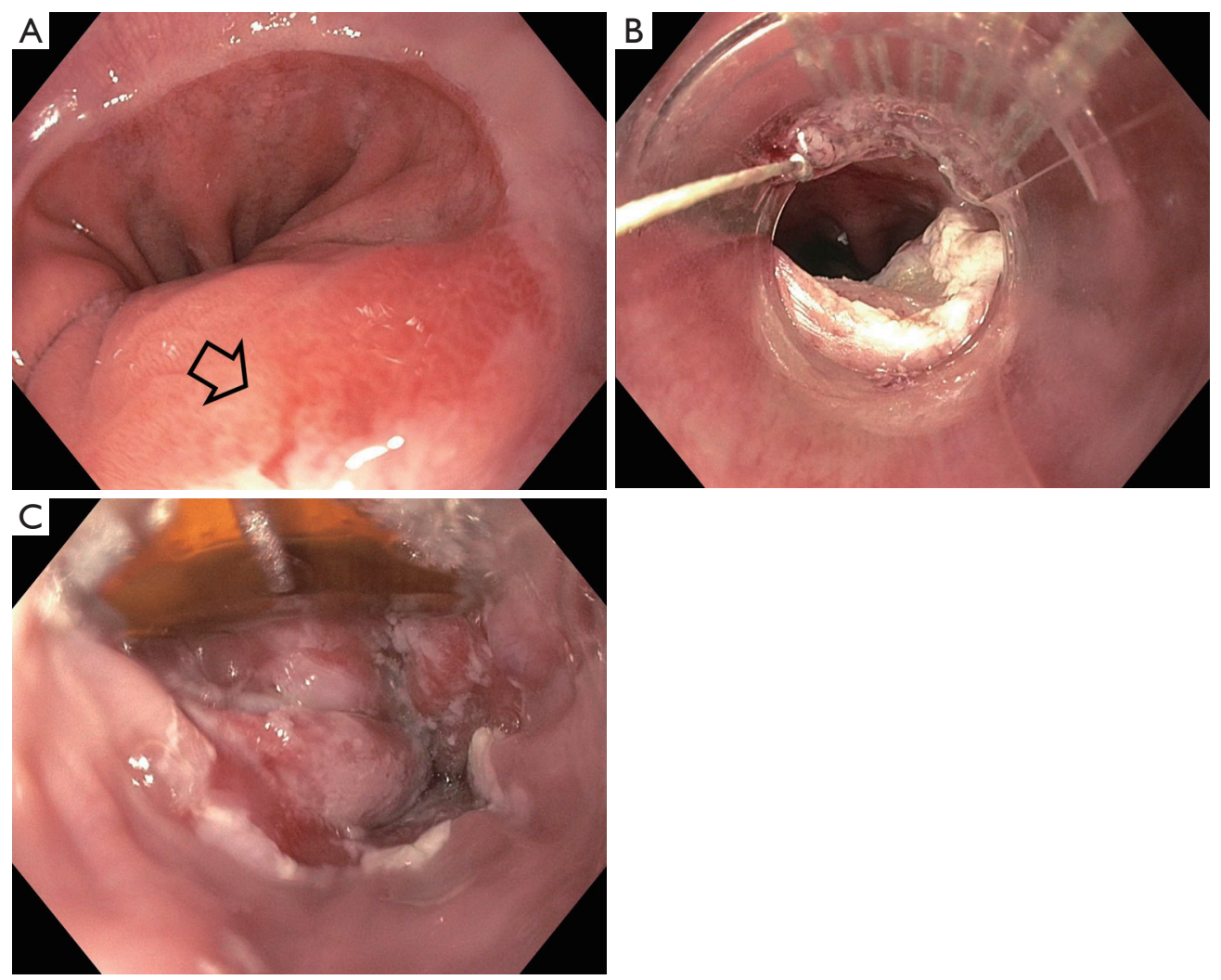

Figure 2 Endoscopic eradication therapy. (A) After careful inspection, a mucosal abnormality (black arrow) was found at the gastroesophageal junction in the background of short segment of Barrett's esophagus (BE). (B) Endoscopic mucosal resection was performed using a bandligation device. Final pathology was consistent with focal high-grade dysplasia containing a minute focus of intramucosal adenocarcinoma (T1a). Lymphovascular invasion not identified. Lateral and deep margins were negative for cancer. (C) On subsequent endoscopy, radiofrequency ablation was performed circumferentially of the short segment BE to achieve complete eradication of intestinal metaplasia.

energy delivered to the esophageal mucosa, inducing tissue necrosis and reformation of the normal esophageal squamous epithelium. The overall safety profile is excellent. In a systematic review, the pooled rate of adverse events from RFA was $8.8 \%$. The rate of esophageal strictures, bleeding, and perforation was measured at a rate of $5.6 \%, 1 \%$ and $0.6 \%$ (52). The RFA catheter can treat the esophagus circumferentially or focally using a balloon or focal catheter device. Typically, circumferential treatment is administered on the initial session and focal ablation is performed during endoscopic follow up to ablate any remaining segments of BE (37). The AIM-Dysplasia trial was the landmark trial that demonstrated a high rate of complete eradication of both dysplasia and intestinal metaplasia. In this multicenter, sham controlled study, 127 patients with dysplastic BE were randomized to RFA or a sham procedure. At 12 months follow up, $91 \%$ of patients with LGD in the ablation group compared with $23 \%$ in the control group $(\mathrm{P}<0.001)$ and $81 \%$ of patients with HGD in the ablation group compared with $19 \%$ in the control group $(\mathrm{P}<0.001)$, achieved complete eradication of dysplasia (CE-D). In addition, the patients in the RFA treatment group had less disease progression $(3.6 \%$ vs. $16.3 \%, \mathrm{P}=0.03)$ and fewer cancers $(1.2 \%$ vs. $9.3 \%, \mathrm{P}=0.045)$ (53). The authors measured the durability of the results in a follow up study and after 3 years, dysplasia was eradicated in $98 \%$ and intestinal metaplasia was eradicated in $91 \%$ of patients (54). In a recent meta-analysis of patients with HGD and IMC, focal EMR followed by RFA had high BE eradication rates with CE-D at $93.4 \%$ and CEIM at $73.1 \%$. In this systematic review, rates of eradication with focal EMR and RFA were comparable to treatment of BE-related neoplasia with solely stepwise EMR and demonstrated a lower rate of stricture formation, bleeding, and perforation (55). 

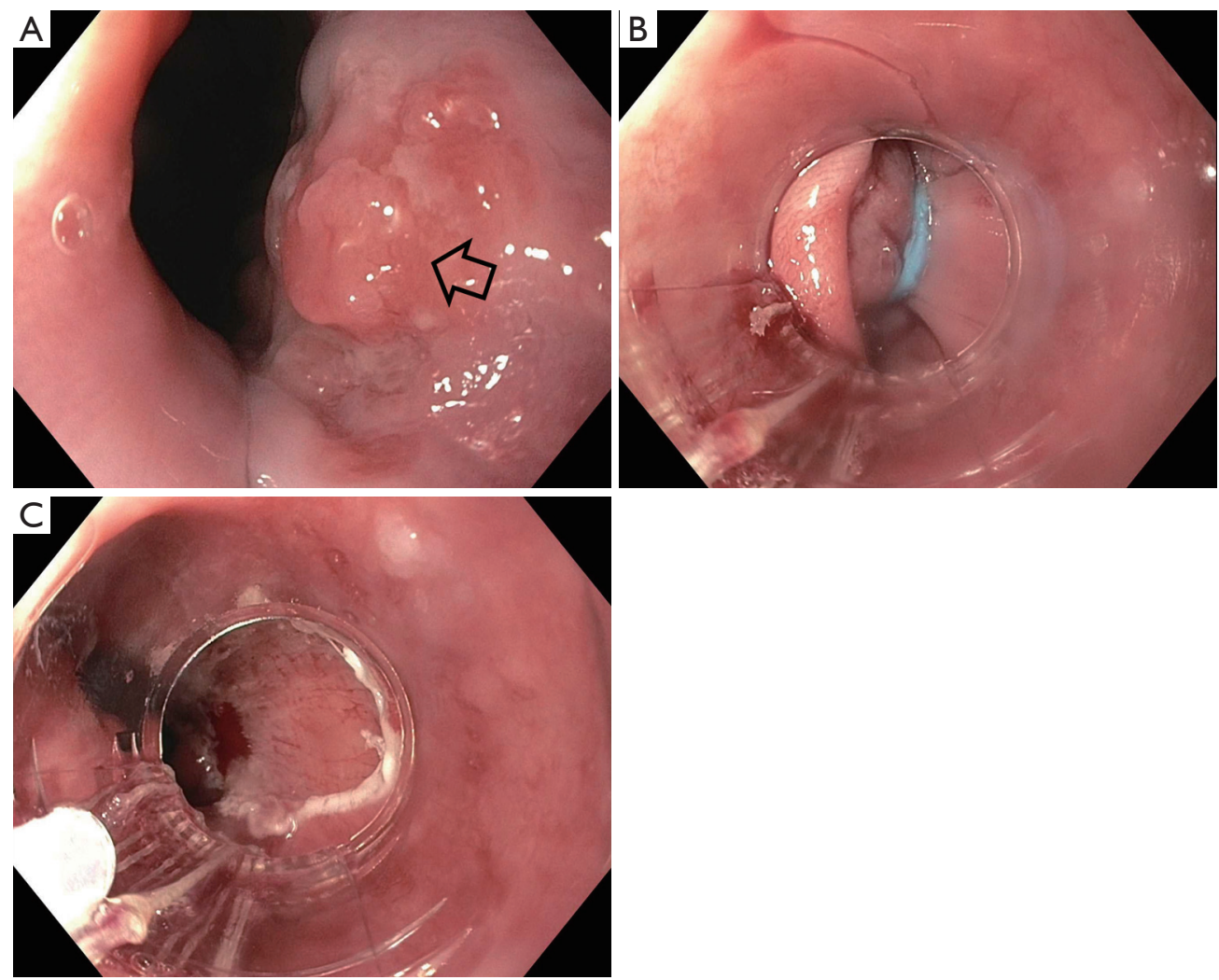

Figure 3 Pathologic staging with endoscopic mucosal resection. (A) After careful inspection, a nodular lesion (black arrow) was identified at the gastroesophageal junction in the background of short segment Barrett's esophagus. (B) Endoscopic mucosal resection was performed using a band-ligation device. The first step in this technique is deploying a band around the lesion and creating a pseudopolyp. (C) Endoscopic resection is then performed using a hot snare. Final pathology revealed invasive adenocarcinoma, moderately to poorly differentiated. The tumor extended to the submucosa (T1b) and lymphovascular invasion was present. Deep and lateral margins were negative for cancer. Given poor pathologic features, patient was referred to surgical oncology.

EET is recommended for confirmed LGD as it reduces the risk of malignant progression. The SURF trial by Phoa et al. was a multicenter trial that enrolled 136 patients with confirmed LGD. In this study, 68 patients were randomized to receive ablation and 68 to receive control. Ablation reduced the risk of progression to HGD or EAC by $25 \%$ (1.5\% for ablation vs. $26.5 \%$ for control; $95 \%$ $\mathrm{CI}, 14.1-35.9 \% ; \mathrm{P}<0.001)$ and the risk of progression to adenocarcinoma by $7.4 \%$ (1.5\% for ablation $v$ s. $8.8 \%$ for control; 95\% CI, 0-14.7\%; $\mathrm{P}=0.03)$. Among patients in the ablation group, CE-D and CEIM occurred in $92 \%$ and $88.2 \%$ compared with $27.9 \%$ and $0 \%$ in the control group $(\mathrm{P}<0.001)$ (56). At 73 months follow up, HGD/EAC was diagnosed in 1 patient in the RFA group (1.5\%) and 23 in the surveillance group (33.8\%) ( $\mathrm{P}=0.000)$, with sustained clearance of BE in $91 \%$ and LGD in $96 \%$ of patients (57).
In a recent meta-analysis of 19 studies, with a total of 2,746 patients, showed a significant reduction in disease progression to HGD/EAC in patients with LGD treated with RFA compared with surveillance (RR 0.14; 95\% CI: 0.04-0.45; $\mathrm{P}=0.001$ ) (58). Current guidelines recommend ablative therapy as the preferred treatment modality for LGD, although endoscopic surveillance every 6-12 months remains an acceptable alternative in certain patients based on patient preference and significant medical comorbidities $(1,37,49,50)$.

Cryotherapy is another emerging technique to treat $\mathrm{BE}$ related neoplasia, and three modalities have been developed. The first system uses carbon dioxide and is administered via a spray catheter. The second technique uses liquid nitrogen via a spray catheter and is the most widely utilized and studied method of cryotherapy. The most recent 
Table 4 Recommendations for endoscopic surveillance of BE associated neoplasia after achieving CEIM with EET

\begin{tabular}{lll}
\hline Histologic grade & \multicolumn{1}{c}{ ACG guidelines (1) } & \multicolumn{1}{c}{ AGA practice update (37) } \\
\hline LGD & Every 6 months for 1 year and annually thereafter & At 1 and 3 years \\
HGD/IMC & $\begin{array}{l}\text { Every } 3 \text { months the } 1^{\text {st }} \text { year, then every } 6 \text { months the } \\
2^{\text {nd }} \text { year, and annually thereafter }\end{array}$ & At 3 months, 6 months, 1 year, and annually thereafter \\
\hline
\end{tabular}

ACG, American College of Gastroenterology; AGA, American Gastrointestinal Association; EET, endoscopic eradication therapy; HGD, high grade dysplasia; IMC, intramucosal adenocarcinoma; LGD, low grade dysplasia; NBDE, non-dysplastic Barrett's esophagus.

device developed utilizes nitrous oxide as its cryogen and is administered by a balloon-based device placed through the endoscope. Cryotherapy involves rapid freezing and thawing of tissue causing immediate and delayed necrosis of the tissue (59-61). A recent meta-analysis was performed to assess the overall efficacy and safety of cryotherapy using liquid nitrogen. Nine studies reported 386 patients who were treated with cryotherapy, the pooled rate of CE-HGD was $86.5 \%$ and CE-IM was $56.5 \%$. In subgroup analysis of patients who failed RFA, the pooled rate of CE-IM was $58.4 \%$ and the pooled rate of CE-D was $81.9 \%$ (62). Another systematic review, also showed cryotherapy successfully achieved CE-D in $76 \%$ of patients who did not respond to initial RFA (63). Hamade et al. evaluated the efficacy as first line treatment in patients BE neoplasia in a systematic review involving 6 studies with a total of 282 patients. The analysis revealed $69 \%$ of the patients treated with cryotherapy achieved CE-IM and $97.9 \%$ had CED. The overall rate of stricture formation was $4.9 \%$ (64). Overall rates of CE-D are comparable to RFA, although further long-term prospective trials are needed.

\section{Post treatment surveillance}

After CEIM has been achieved with EET, post treatment surveillance intervals are based on the original histologic diagnosis (Table 4). Recently, the AGA published a practice update and recommended an attenuated surveillance approach from prior guidelines. In patients with HGD, surveillance endoscopy can be performed at 3, 6, and 12 months and annually thereafter. In patients with LGD, surveillance endoscopy can be performed at 1 and 3 years $(37,65)$.

Experts define recurrence as the presence of IM, dysplasia or EAC in the tubular esophagus or GEJ after successful EET (29). One meta-analysis reported rates of recurrent IM, dysplastic BE, and HGD/EAC after RFA at 9.5\%, 2.0\% and $1.2 \%$ per patient-years (30). A recent prospective study of 807 patients with BE (majority with HGD/IMC), who underwent EET, reported a recurrence of IM in $15 \%$ for an incidence rate of 5.2 per 100 person years, and recurrence of dysplasia in $5.1 \%$ for an incidence rate of 1.8 per 100 person years (31). In contrast to previous studies which demonstrated highest recurrence in the first year $(66,67)$, in this study, Wani et al. reported the risk of recurrence was highest between $1^{\text {st }}$ and $2^{\text {nd }}$ year of follow up (31).

Risk factors for recurrence have been addressed in multiple studies. In a meta-analysis, predictors of recurrence were age and BE length (30). In a recent prospective study, additional predictors associated with recurrence included high grade dysplasia or intramucosal cancer, presence of reflux symptoms or hiatal hernia, and increased number of sessions required to achieve CEIM (31).

Current guidelines for post therapy endoscopic surveillance involves 4-quadrant biopsies every 1-2 cm throughout the length of the original Barrett's segment and the gastric cardia (37). The location of recurrence has been evaluated and the majority of recurrences were detected in the distal $2 \mathrm{~cm}$ of the esophagus (68). Another study found $74 \%$ of BE recurrences at the GE junction and $26 \%$ in the tubular esophagus, with the majority found within $5 \mathrm{~cm}$ of the GEJ (69). Based on current evidence, experts proposed a modified approach with targeted biopsies of any visible abnormalities and random four-quadrant biopsies more geared towards the distal esophagus and region of the GEJ and gastric cardia $(29,32,68)$.

Recurrences are treated similarly to initial treatment protocols involving EMR, ESD or ablative modalities. Antireflux therapy is recommended to achieve symptoms control and absence of erosive esophagitis.

\section{Molecular alterations in $B E$}

Histologically, NDBE can progress from low to high grade dysplasia, and ultimately invasive carcinoma. The frequency of endoscopic surveillance and clinical management of $\mathrm{BE}$ is based on the grade of dysplasia. Current recommendations rely only on histologic data from biopsies or resection specimens to risk stratify patients. The limitations to the 
current strategies include difficulties with identifying dysplasia endoscopically, sampling error during surveillance biopsies and low interobserver reproducibility in the diagnosis of dysplasia among pathologists (9). To overcome these challenges, significant interest has been made in further understanding the molecular factors associated with progression of $\mathrm{BE}$ and to develop predictive markers to aid in diagnosis and risk stratification.

The progression from non-dysplastic BE to EAC involves several molecular changes. These changes include structural genomic alterations, DNA sequence alterations, and epigenetic modifications (70). Genomic profiles studied in patients with more progressive disease have demonstrated increasing chromosomal instability, genomic diversity and genomic doubling (71). The accumulation of these genetic modifications drives the development of neoplasia and progression of the disease process.

Early studies have evaluated specific genetic anomalies in $\mathrm{BE}$ and EAC. Loss of heterozygosity (LOH) at $17 \mathrm{p}$ and $9 \mathrm{p}$ has been found in BE and EAC patients and these loci harbor the tumor suppressor genes TP53 and CKDN2A (72). Inactivation of tumor suppressor genes lead to genomic instability and uncontrolled cell multiplication. Approximately $80 \%$ of $\mathrm{BE}$ is associated with anomalies in CKDN2A and can arise from $\mathrm{LOH}$ or hypermethylation of the promoter sequence of CKDN2A (73). Various studies have shown alteration of CKDN2A is an initiating event in the pathogenesis of $\mathrm{BE}(72)$.

TP53 abnormalities have been strongly associated with neoplastic progression in BE $(74,75)$. TP53 protein accumulation detected by immunohistochemistry (IHC) indirectly assesses TP53 mutations. Davelaar et al. combined techniques in IHC and DNA fluorescent in situ hybridization (FISH) to increase detection of TP53 abnormalities in BE patients. Multivariate analyses showed that abnormalities in TP53 on IHC and FISH were independent predictors of progression (76). Younes and colleagues prospectively followed 275 patients with BE with no HGD or EAC for 41 months, and found p53 accumulation, detected by IHC was a significant predictor of malignant progression to HGD or EAC (77). Kastelein and colleagues also showed a complete absent pattern of p53 expression was also associated with higher risk of neoplastic progression (75). A recent meta-analysis describing 8 case-control studies comprising 1,435 patients and 7 cohort studies comprising 582 patients found strong, and significant associations between aberrant p53 immunostaining and progression to high-grade dysplasia or esophageal adenocarcinoma (78).

Genomic alterations and instability have been associated with the pathogenesis of $\mathrm{BE}$ neoplasia in numerous studies. Aneuploidy is defined as the presence of an abnormal number of chromosomes in a cell. Image cytometric DNA analysis is a method to examine DNA content abnormalities, and in early studies has shown to be a predictor of progression to EAC and HGD in patients with BE (79). Recently Choi and colleagues analyzed 139 BE biopsy samples from 124 patients with varying degrees of dysplasia. DNA content abnormality detected by flow cytometry was identified in $95 \%$ of HGD samples. In addition, $87.5 \%$ of LGD patients with DNA content abnormality were subsequently found to have HGD or EAC within a year, whereas only $16.7 \%$ of patients with LGD in the setting of normal flow cytometric results developed HGD or EAC (80).

Epigenetic modifications have shown to play a significant role in progression of BE. Aberrant DNA methylation is the most studied epigenetic modification in cancer pathogenesis and the mechanism involves methylation of cytosine bases in CG-rich sequences, called CPG islands (70). One of the first tumor suppressor genes shown to be aberrantly methylated in BE was CDKN2A. Several studies have evaluated hypermethylation of several other genes involved in the development of BE neoplasia and EAC (81-88). Furthermore, Kaz et al. found various histologic groups (normal squamous esophagus, NDBE, HGD, EAC) had distinct methylation signatures and demonstrated increasing methylation progressing from normal squamous epithelium to $\mathrm{BE}$ to HGD/EAC. In addition, the authors discovered high and low methylation epigenotypes within the BE and EAC cases (89).

MircoRNAs have also demonstrated to play a role in various cancers and the pathogenesis of $\mathrm{BE}$ and $\mathrm{EAC}$. MiRNAs are small noncoding RNA molecules that exert their influence at the time of post transcription resulting in regulation of tumor suppressor or oncogenes $(72,90) . \mathrm{Li}$ et al. analyzed miRNA expression patterns in esophageal tissues collected by Cytosponge and found a panel of 11 miRNAs up-regulated in BE patients (91). Several studies have also found specific alterations in miRNA expressions associated with progression BE and EAC $(90,92)$.

The development of next generation sequencing (NGS) technology has improved our understanding of the complex molecular relationship between BE and EAC. In an early NGS study, Dulak et al. identified 26 genes with significantly recurring mutations $\mathrm{n}$ EAC. The authors found TP53 and CDKNA2A were the most frequently 
mutated genes in EAC (93). A large sequencing study found that common mutated genes in EAC were also mutated in NDBE, including tumor suppressors such as CDKN2A and ARID1A. Only mutations in TP53 were found in HGD and EAC, and SMAD4 were detected only in EAC (94). More recently, Stachler and colleagues performed genomic analyses on 24 patients who were under routine $\mathrm{BE}$ surveillance and later progressed to HGD or EAC more than 1 year after index BE diagnosis and compared them to matched controls. In this study, TP53 mutations were detected in $46 \%$ of samples from progressors and $5 \%$ of non-progressors. TP53 mutations increased the risk of progression 13.8-fold (95\% CI, 3.2-61, $\mathrm{P}<0.001$ ) supporting its role as a substantial genomic marker in BE neoplastic progression. In contrast to previous studies, PT53 mutations were also detected in NDBE and identified patients at increased risk for progression (95).

Several studies have shown multiple other tumor suppressor genes and oncogenes that have been implicated in the progression of BE to EAC. These genes are involved in mechanisms of DNA repair, cell cycle, cell adhesion, detoxification of reactive oxygen species, vascularization, reprograming of energy metabolism and various signaling pathways involving tyrosine kinases, Wnt/ $\beta$-catenin, Notch, and Hedgehog signaling (95-97).

These molecular studies demonstrate the complex mutational landscape found in EAC as well as in NDBE, and may explain an alternative model to the traditional stepwise theory of cancer development (94). The alternative model suggests early in the disease process, there is an accrual of mutations that may play a role in clonal expansion and diversity, and as necessary driver mutations have been acquired, lead to genomic instability and an accelerated progression to EAC (70).

\section{Biomarkers}

Several biomarkers have shown promise to improve risk stratification of patients with $\mathrm{BE}$. Biomarker panels aim to incorporate the molecular heterogeneity involved in the progression of $\mathrm{BE}$ and target a combination of genomic/ structural alterations, epigenetic markers, and miRNA expression to improve sensitivity. In addition, other protein markers have been evaluated including specific glycanbinding proteins called lectins. Bird-Lieberman et al. described a panel comprising of LGD, abnormal DNA ploidy, and Aspergillus oryzae lectin (AOL) that accurately identified $\mathrm{BE}$ patients at higher risk for progression to
HGD or EAC (98). Recently, Duits et al. found that an expert diagnosis of LGD, abnormal p53 expression and abnormal expression of AOL were independently associated with progression to HGD/EAC (99). Ross-Innes et al. combined the use of a non-endoscopic sampling device and a multidimensional biomarker panel (p53 mutational status, various protein markers, methylation markers, presence of glandular atypia) and identified with a high level of confidence patients with BE who are dysplasia free (100). Numerous markers are currently being investigated and while research is still ongoing, may prove to be a tool to use in adjunct to clinical prediction scores and current diagnostic and surveillance strategies.

\section{Conclusions}

$\mathrm{BE}$ is the only established precursor lesion to esophageal adenocarcinoma and can progress in a stepwise fashion from NDBE to LGD and HGD, and ultimately invasive carcinoma. Substantial progress has been made on the treatment of dysplastic BE and early esophageal cancer with endoscopic techniques. The combination of EMR and RFA for BE neoplasia have demonstrated high eradication rates for dysplasia, intestinal metaplasia, and reduced risk of progression to EAC. Newer emerging endoscopic techniques have shown good outcomes and include ESD for resection of larger and bulkier lesions, and cryotherapy as an alternative ablative strategy for patient's refractory to RFA or even as first line therapy as the evidence evolves. Despite effective treatment strategies, our current model for screening and surveillance has not impacted the rising incidence of EAC in Western countries. This has led to significant breakthroughs in our understanding of the molecular aspects of BE and EAC. The progression of $\mathrm{BE}$ to EAC involves a significant number of molecular changes involving genetic and epigenetic modifications. Genome-wide next generation sequencing techniques has significantly added to the understanding of the numerous somatic DNA aberrations that occur even at an early stage of BE pathogenesis and suggests a more complex model to esophageal cancer development than previously thought. Multiple biomarkers and minimally invasive nonendoscopic screening tools are being investigated to aid in the diagnosis of BE with early promising results and randomized control trials are currently underway. Further studies are still needed to develop and refine markers that identify patients at highest risk for progression to HGD or EAC at an early stage in the disease process. 


\section{Acknowledgments}

Funding: None.

\section{Footnote}

Provenance and Peer Review: This article was commissioned by the Guest Editor (Dr. Khaldoun Almhanna) for the series "Gastroesophageal Cancer 2020" published in Annals of Translational Medicine. The article was sent for external peer review organized by the Guest Editor and the editorial office.

Reporting Checklist: The authors have completed the Narrative Review reporting checklist. Available at http:// dx.doi.org/10.21037/atm-20-4406

Conflicts of Interest: Both authors have completed the ICMJE uniform disclosure form (available at http://dx.doi. org/10.21037/atm-20-4406). The series "Gastroesophageal Cancer 2020" was commissioned by the editorial office without any funding or sponsorship. The authors have no other conflicts of interest to declare.

Ethical Statement: The authors are accountable for all aspects of the work in ensuring that questions related to the accuracy or integrity of any part of the work are appropriately investigated and resolved.

Open Access Statement: This is an Open Access article distributed in accordance with the Creative Commons Attribution-NonCommercial-NoDerivs 4.0 International License (CC BY-NC-ND 4.0), which permits the noncommercial replication and distribution of the article with the strict proviso that no changes or edits are made and the original work is properly cited (including links to both the formal publication through the relevant DOI and the license). See: https://creativecommons.org/licenses/by-nc-nd/4.0/.

\section{References}

1. Shaheen NJ, Falk GW, Iyer PG, et al. ACG Clinical Guideline: Diagnosis and Management of Barrett's Esophagus. Am J Gastroenterol 2016;111:30-50; quiz 1.

2. Ronkainen J, Aro P, Storskrubb T, et al. Prevalence of Barrett's esophagus in the general population: an endoscopic study. Gastroenterology 2005;129:1825-31.

3. Fan X, Snyder N. Prevalence of Barrett's esophagus in patients with or without GERD symptoms: role of race, age, and gender. Dig Dis Sci 2009;54:572-7.

4. Lin EC, Holub J, Lieberman D, et al. Low Prevalence of Suspected Barrett's Esophagus in Patients With Gastroesophageal Reflux Disease Without Alarm Symptoms. Clin Gastroenterol Hepatol 2019;17:857-63.

5. Qumseya BJ, Bukannan A, Gendy S, et al. Systematic review and meta-analysis of prevalence and risk factors for Barrett's esophagus. Gastrointest Endosc 2019;90:707-17.e1.

6. Bhat S, Coleman HG, Yousef F, et al. Risk of malignant progression in Barrett's esophagus patients: results from a large population-based study. J Natl Cancer Inst 2011;103:1049-57.

7. Kelty C. Columnar-lined esophagus without intestinal metaplasia should not be dismissed as having no cancer risk. Am J Gastroenterol 2008;103:3205; author reply -6.

8. Harrison R, Perry I, Haddadin W, et al. Detection of intestinal metaplasia in Barrett's esophagus: an observational comparator study suggests the need for a minimum of eight biopsies. Am J Gastroenterol 2007;102:1154-61.

9. Eluri S, Shaheen NJ. Barrett's esophagus: diagnosis and management. Gastrointest Endosc 2017;85:889-903.

10. Anaparthy R, Gaddam S, Kanakadandi V, et al. Association between length of Barrett's esophagus and risk of highgrade dysplasia or adenocarcinoma in patients without dysplasia. Clin Gastroenterol Hepatol 2013;11:1430-6.

11. Hur C, Miller M, Kong CY, et al. Trends in esophageal adenocarcinoma incidence and mortality. Cancer 2013;119:1149-58.

12. Bhat SK, McManus DT, Coleman HG, et al. Oesophageal adenocarcinoma and prior diagnosis of Barrett's oesophagus: a population-based study. Gut 2015;64:20-5.

13. Otaki F, Iyer PG. Point-Counterpoint: Screening and Surveillance for Barrett's Esophagus, Is It Worthwhile? Dig Dis Sci 2018;63:2081-93.

14. ASGE Standards of Practice Committee, Ben-Menachem T, Decker GA, et al. Adverse events of upper GI endoscopy. Gastrointest Endosc 2012;76:707-18.

15. Visrodia K, Singh S, Krishnamoorthi R, et al. Magnitude of Missed Esophageal Adenocarcinoma After Barrett's Esophagus Diagnosis: A Systematic Review and Meta-analysis. Gastroenterology 2016;150:599-607. e7; quiz e14-5.

16. Visrodia K, Iyer PG, Schleck CD, et al. Yield of Repeat Endoscopy in Barrett's Esophagus with No Dysplasia and Low-Grade Dysplasia: A Population-Based Study. Dig Dis 
Sci 2016;61:158-67.

17. Codipilly DC, Iyer PG. Novel Screening Tests for Barrett's Esophagus. Curr Gastroenterol Rep 2019;21:42.

18. Spechler SJ, Katzka DA, Fitzgerald RC. New Screening Techniques in Barrett's Esophagus: Great Ideas or Great Practice? Gastroenterology 2018;154:1594-601.

19. Ross-Innes CS, Debiram-Beecham I, O'Donovan M, et al. Evaluation of a minimally invasive cell sampling device coupled with assessment of trefoil factor 3 expression for diagnosing Barrett's esophagus: a multi-center casecontrol study. PLoS Med 2015;12:e1001780.

20. Kadri SR, Lao-Sirieix P, O'Donovan M, et al. Acceptability and accuracy of a non-endoscopic screening test for Barrett's oesophagus in primary care: cohort study. BMJ 2010;341:c4372.

21. Offman J, Muldrew B, O'Donovan M, et al. Barrett's oESophagus trial 3 (BEST3): study protocol for a randomised controlled trial comparing the CytospongeTFF3 test with usual care to facilitate the diagnosis of oesophageal pre-cancer in primary care patients with chronic acid reflux. BMC Cancer 2018;18:784.

22. Moinova HR, LaFramboise T, Lutterbaugh JD, et al. Identifying DNA methylation biomarkers for nonendoscopic detection of Barrett's esophagus. Sci Transl Med 2018;10:eaao5848.

23. Iyer PG, Taylor WR, Johnson ML, et al. Highly Discriminant Methylated DNA Markers for the Nonendoscopic Detection of Barrett's Esophagus. Am J Gastroenterol 2018;113:1156-66.

24. Wang Z, Kambhampati S, Cheng Y, et al. Methylation Biomarker Panel Performance in EsophaCap Cytology Samples for Diagnosing Barrett's Esophagus: A Prospective Validation Study. Clin Cancer Res 2019;25:2127-35.

25. Chettouh H, Mowforth O, Galeano-Dalmau N, et al. Methylation panel is a diagnostic biomarker for Barrett's oesophagus in endoscopic biopsies and non-endoscopic cytology specimens. Gut 2018;67:1942-9.

26. Chan DK, Zakko L, Visrodia KH, et al. Breath Testing for Barrett's Esophagus Using Exhaled Volatile Organic Compound Profiling With an Electronic Nose Device. Gastroenterology 2017;152:24-6.

27. Krishnamoorthi R, Singh S, Ragunathan K, et al. Factors Associated With Progression of Barrett's Esophagus: A Systematic Review and Meta-analysis. Clin Gastroenterol Hepatol 2018;16:1046-55.e8.

28. Parasa S, Vennalaganti S, Gaddam S, et al. Development and Validation of a Model to Determine Risk of Progression of Barrett's Esophagus to Neoplasia.
Gastroenterology 2018;154:1282-9.e2.

29. Kahn A, Shaheen NJ, Iyer PG. Approach to the PostAblation Barrett's Esophagus Patient. Am J Gastroenterol 2020;115:823-31.

30. Krishnamoorthi R, Singh S, Ragunathan K, et al. Risk of recurrence of Barrett's esophagus after successful endoscopic therapy. Gastrointest Endosc 2016;83:1090106.e3.

31. Wani S, Han S, Kushnir V, et al. Recurrence Is Rare Following Complete Eradication of Intestinal Metaplasia in Patients With Barrett's Esophagus and Peaks at 18 Months. Clin Gastroenterol Hepatol 2020. [Epub ahead of print].

32. Reed CC, Shaheen NJ. Management of Barrett Esophagus Following Radiofrequency Ablation. Gastroenterol Hepatol (N Y) 2019;15:377-86.

33. Duits LC, Phoa KN, Curvers WL, et al. Barrett's oesophagus patients with low-grade dysplasia can be accurately risk-stratified after histological review by an expert pathology panel. Gut 2015;64:700-6.

34. Curvers WL, ten Kate FJ, Krishnadath KK, et al. Lowgrade dysplasia in Barrett's esophagus: overdiagnosed and underestimated. Am J Gastroenterol 2010;105:1523-30.

35. Duits LC, van der Wel MJ, Cotton CC, et al. Patients With Barrett's Esophagus and Confirmed Persistent LowGrade Dysplasia Are at Increased Risk for Progression to Neoplasia. Gastroenterology 2017;152:993-1001.e1.

36. Kestens C, Offerhaus GJ, van Baal JW, et al. Patients With Barrett's Esophagus and Persistent Low-grade Dysplasia Have an Increased Risk for High-grade Dysplasia and Cancer. Clin Gastroenterol Hepatol 2016;14:956-62.e1.

37. Sharma P, Shaheen NJ, Katzka D, et al. AGA Clinical Practice Update on Endoscopic Treatment of Barrett's Esophagus With Dysplasia and/or Early Cancer: Expert Review. Gastroenterology 2020;158:760-9.

38. Rastogi A, Puli S, El-Serag HB, et al. Incidence of esophageal adenocarcinoma in patients with Barrett's esophagus and high-grade dysplasia: a meta-analysis. Gastrointest Endosc 2008;67:394-8.

39. Codipilly DC, Chandar AK, Singh S, et al. The Effect of Endoscopic Surveillance in Patients With Barrett's Esophagus: A Systematic Review and Meta-analysis. Gastroenterology 2018;154:2068-86.e5.

40. Wenker TN, Tan MC, Liu Y, et al. Prior Diagnosis of Barrett's Esophagus Is Infrequent, but Associated with Improved Esophageal Adenocarcinoma Survival. Dig Dis Sci 2018;63:3112-9.

41. Tramontano AC, Sheehan DF, Yeh JM, et al. The 
Impact of a Prior Diagnosis of Barrett's Esophagus on Esophageal Adenocarcinoma Survival. Am J Gastroenterol 2017;112:1256-64.

42. Kerkhof M, van Dekken H, Steyerberg EW, et al. Grading of dysplasia in Barrett's oesophagus: substantial interobserver variation between general and gastrointestinal pathologists. Histopathology 2007;50:920-7.

43. Peters FP, Brakenhoff KP, Curvers WL, et al. Histologic evaluation of resection specimens obtained at 293 endoscopic resections in Barrett's esophagus. Gastrointest Endosc 2008;67:604-9.

44. Smith MS, Ikonomi E, Bhuta R, et al. Wide-area transepithelial sampling with computer-assisted 3-dimensional analysis (WATS) markedly improves detection of esophageal dysplasia and Barrett's esophagus: analysis from a prospective multicenter community-based study. Dis Esophagus 2019;32:doy099.

45. ASGE Standards of Practice Committee, Qumseya B, Sultan S, et al. ASGE guideline on screening and surveillance of Barrett's esophagus. Gastrointest Endosc 2019;90:335-359.e2.

46. Qumseya BJ, Wang H, Badie N, et al. Advanced imaging technologies increase detection of dysplasia and neoplasia in patients with Barrett's esophagus: a meta-analysis and systematic review. Clin Gastroenterol Hepatol 2013;11:1562-70.e1-2.

47. Gorrepati VS, Sharma P. How Should We Report Endoscopic Results in Patient's with Barrett's Esophagus? Dig Dis Sci 2018;63:2115-21.

48. Sharma P, Savides TJ, Canto MI, et al. The American Society for Gastrointestinal Endoscopy PIVI (Preservation and Incorporation of Valuable Endoscopic Innovations) on imaging in Barrett's Esophagus. Gastrointest Endosc 2012;76:252-4.

49. Wani S, Rubenstein JH, Vieth M, et al. Diagnosis and Management of Low-Grade Dysplasia in Barrett's Esophagus: Expert Review From the Clinical Practice Updates Committee of the American Gastroenterological Association. Gastroenterology 2016;151:822-35.

50. Standards of Practice Committee, Wani S, Qumseya B, et al. Endoscopic eradication therapy for patients with Barrett's esophagus-associated dysplasia and intramucosal cancer. Gastrointest Endosc 2018;87:907-931.e9.

51. Peters Y, Al-Kaabi A, Shaheen NJ, et al. Barrett oesophagus. Nat Rev Dis Primers 2019;5:35.

52. Qumseya BJ, Wani S, Desai M, et al. Adverse Events After Radiofrequency Ablation in Patients With Barrett's
Esophagus: A Systematic Review and Meta-analysis. Clin Gastroenterol Hepatol 2016;14:1086-95.e6.

53. Shaheen NJ, Sharma P, Overholt BF, et al. Radiofrequency ablation in Barrett's esophagus with dysplasia. N Engl J Med 2009;360:2277-88.

54. Shaheen NJ, Overholt BF, Sampliner RE, et al. Durability of radiofrequency ablation in Barrett's esophagus with dysplasia. Gastroenterology 2011;141:460-8.

55. Desai M, Saligram S, Gupta N, et al. Efficacy and safety outcomes of multimodal endoscopic eradication therapy in Barrett's esophagus-related neoplasia: a systematic review and pooled analysis. Gastrointest Endosc 2017;85:482-95.e4.

56. Phoa KN, van Vilsteren FG, Weusten BL, et al. Radiofrequency ablation vs endoscopic surveillance for patients with Barrett esophagus and low-grade dysplasia: a randomized clinical trial. JAMA 2014;311:1209-17.

57. Pouw RE, Klaver E, Phoa KN, et al. Radiofrequency ablation for low-grade dysplasia in Barrett's esophagus: long-term outcome of a randomized trial. Gastrointest Endosc 2020. [Epub ahead of print].

58. Qumseya BJ, Wani S, Gendy S, et al. Disease Progression in Barrett's Low-Grade Dysplasia With Radiofrequency Ablation Compared With Surveillance: Systematic Review and Meta-Analysis. Am J Gastroenterol 2017;112:849-65.

59. Canto MI. Cryotherapy for Barrett's Esophagus. Gastrointest Endosc Clin N Am 2017;27:503-13.

60. Overwater A, Weusten B. Cryoablation in the management of Barrett's esophagus. Curr Opin Gastroenterol 2017;33:261-9.

61. Visrodia K, Zakko L, Wang KK. Mucosal Ablation in Patients with Barrett's Esophagus: Fry or Freeze? Dig Dis Sci 2018;63:2129-35.

62. Mohan BP, Krishnamoorthi R, Ponnada S, et al. Liquid Nitrogen Spray Cryotherapy in Treatment of Barrett's Esophagus, where do we stand? A Systematic Review and Meta-Analysis. Dis Esophagus 2019;32:doy130.

63. Visrodia K, Zakko L, Singh S, et al. Cryotherapy for persistent Barrett's esophagus after radiofrequency ablation: a systematic review and meta-analysis. Gastrointest Endosc 2018;87:1396-404.e1.

64. Hamade N, Desai M, Thoguluva Chandrasekar V, et al. Efficacy of cryotherapy as first line therapy in patients with Barrett's neoplasia: a systematic review and pooled analysis. Dis Esophagus 2019;32:doz040.

65. Cotton CC, Haidry R, Thrift AP, et al. Development of Evidence-Based Surveillance Intervals After Radiofrequency Ablation of Barrett's Esophagus. 
Gastroenterology 2018;155:316-26.e6.

66. Cotton CC, Wolf WA, Overholt BF, et al. Late Recurrence of Barrett's Esophagus After Complete Eradication of Intestinal Metaplasia is Rare: Final Report From Ablation in Intestinal Metaplasia Containing Dysplasia Trial. Gastroenterology 2017;153:681-8.e2.

67. Sawas T, Iyer PG, Alsawas M, et al. Higher Rate of Barrett's Detection in the First Year After Successful Endoscopic Therapy: Meta-analysis. Am J Gastroenterol 2018;113:959-71.

68. Omar M, Thaker AM, Wani S, et al. Anatomic location of Barrett's esophagus recurrence after endoscopic eradication therapy: development of a simplified surveillance biopsy strategy. Gastrointest Endosc 2019;90:395-403.

69. Sami SS, Ravindran A, Kahn A, et al. Timeline and location of recurrence following successful ablation in Barrett's oesophagus: an international multicentre study. Gut 2019;68:1379-85.

70. Grady WM, Yu M. Molecular Evolution of Metaplasia to Adenocarcinoma in the Esophagus. Dig Dis Sci 2018;63:2059-69.

71. Li X, Galipeau PC, Paulson TG, et al. Temporal and spatial evolution of somatic chromosomal alterations: a case-cohort study of Barrett's esophagus. Cancer Prev Res (Phila) 2014;7:114-27.

72. Kaz AM, Grady WM, Stachler MD, et al. Genetic and Epigenetic Alterations in Barrett's Esophagus and Esophageal Adenocarcinoma. Gastroenterol Clin North Am 2015;44:473-89.

73. Mudyanadzo TA. Barrett's Esophagus: A Molecular Overview. Cureus 2018;10:e3468.

74. Murray L, Sedo A, Scott M, et al. TP53 and progression from Barrett's metaplasia to oesophageal adenocarcinoma in a UK population cohort. Gut 2006;55:1390-7.

75. Kastelein F, Biermann K, Steyerberg EW, et al. Aberrant p53 protein expression is associated with an increased risk of neoplastic progression in patients with Barrett's oesophagus. Gut 2013;62:1676-83.

76. Davelaar AL, Calpe S, Lau L, et al. Aberrant TP53 detected by combining immunohistochemistry and DNAFISH improves Barrett's esophagus progression prediction: a prospective follow-up study. Genes Chromosomes Cancer 2015;54:82-90.

77. Younes M, Brown K, Lauwers GY, et al. p53 protein accumulation predicts malignant progression in Barrett's metaplasia: a prospective study of 275 patients. Histopathology 2017;71:27-33.

78. Snyder P, Dunbar K, Cipher DJ, et al. Aberrant p53
Immunostaining in Barrett's Esophagus Predicts Neoplastic Progression: Systematic Review and MetaAnalyses. Dig Dis Sci 2019;64:1089-97.

79. Fang M, Lew E, Klein M, et al. DNA abnormalities as marker of risk for progression of Barrett's esophagus to adenocarcinoma: image cytometric DNA analysis in formalin-fixed tissues. Am J Gastroenterol 2004;99:1887-94.

80. Choi WT, Tsai JH, Rabinovitch PS, et al. Diagnosis and risk stratification of Barrett's dysplasia by flow cytometric DNA analysis of paraffin-embedded tissue. Gut 2018;67:1229-38.

81. Smith E, De Young NJ, Pavey SJ, et al. Similarity of aberrant DNA methylation in Barrett's esophagus and esophageal adenocarcinoma. Mol Cancer 2008;7:75.

82. Schulmann K, Sterian A, Berki A, et al. Inactivation of p16, RUNX3, and HPP1 occurs early in Barrett'sassociated neoplastic progression and predicts progression risk. Oncogene 2005;24:4138-48.

83. Tischoff I, Hengge UR, Vieth M, et al. Methylation of SOCS-3 and SOCS-1 in the carcinogenesis of Barrett's adenocarcinoma. Gut 2007;56:1047-53.

84. Jin Z, Cheng Y, Gu W, et al. A multicenter, doubleblinded validation study of methylation biomarkers for progression prediction in Barrett's esophagus. Cancer Res 2009;69:4112-5.

85. Alvi MA, Liu X, O'Donovan M, et al. DNA methylation as an adjunct to histopathology to detect prevalent, inconspicuous dysplasia and early-stage neoplasia in Barrett's esophagus. Clin Cancer Res 2013;19:878-88.

86. Clément G, Braunschweig R, Pasquier N, et al. Methylation of APC, TIMP3, and TERT: a new predictive marker to distinguish Barrett's oesophagus patients at risk for malignant transformation. J Pathol 2006;208:100-7.

87. Wang JS, Guo M, Montgomery EA, et al. DNA promoter hypermethylation of p16 and APC predicts neoplastic progression in Barrett's esophagus. Am J Gastroenterol 2009;104:2153-60.

88. Kuester D, El-Rifai W, Peng D, et al. Silencing of MGMT expression by promoter hypermethylation in the metaplasia-dysplasia-carcinoma sequence of Barrett's esophagus. Cancer Lett 2009;275:117-26.

89. Kaz AM, Wong CJ, Luo Y, et al. DNA methylation profiling in Barrett's esophagus and esophageal adenocarcinoma reveals unique methylation signatures and molecular subclasses. Epigenetics 2011;6:1403-12.

90. Kong X, Gong S, Su L, et al. Expression signatures and roles of MicroRNAs in human oesophageal 


\section{Page 16 of 16}

adenocarcinomas. J Cell Mol Med 2018;22:123-30.

91. Li X, Kleeman S, Coburn SB, et al. Selection and Application of Tissue microRNAs for Nonendoscopic Diagnosis of Barrett's Esophagus. Gastroenterology 2018;155:771-83.e3.

92. Craig MP, Rajakaruna S, Paliy O, et al. Differential MicroRNA Signatures in the Pathogenesis of Barrett's Esophagus. Clin Transl Gastroenterol 2020;11:e00125.

93. Dulak AM, Stojanov P, Peng S, et al. Exome and wholegenome sequencing of esophageal adenocarcinoma identifies recurrent driver events and mutational complexity. Nat Genet 2013;45:478-86.

94. Weaver JMJ, Ross-Innes CS, Shannon N, et al. Ordering of mutations in preinvasive disease stages of esophageal carcinogenesis. Nat Genet 2014;46:837-43.

95. Stachler MD, Camarda ND, Deitrick C, et al. Detection of Mutations in Barrett's Esophagus Before Progression to High-Grade Dysplasia or Adenocarcinoma. Gastroenterology 2018;155:156-67.

96. Kalatskaya I. Overview of major molecular alterations

Cite this article as: Dam AN, Klapman J. A narrative review of Barrett's esophagus in 2020, molecular and clinical update. Ann Transl Med 2020;8(17):1107. doi: 10.21037/atm-20-4406
Dam and Klapman. BE in 2020, molecular and clinical update

during progression from Barrett's esophagus to esophageal adenocarcinoma. Ann N Y Acad Sci 2016;1381:74-91.

97. Caspa Gokulan R, Garcia-Buitrago MT, Zaika AI. From genetics to signaling pathways: molecular pathogenesis of esophageal adenocarcinoma. Biochim Biophys Acta Rev Cancer 2019;1872:37-48.

98. Bird-Lieberman EL, Dunn JM, Coleman HG, et al. Population-based study reveals new risk-stratification biomarker panel for Barrett's esophagus. Gastroenterology 2012;143:927-35 e3.

99. Duits LC, Lao-Sirieix P, Wolf WA, et al. A biomarker panel predicts progression of Barrett's esophagus to esophageal adenocarcinoma. Dis Esophagus 2019;32:doy102.

100. Ross-Innes CS, Chettouh H, Achilleos A, et al. Risk stratification of Barrett's oesophagus using a nonendoscopic sampling method coupled with a biomarker panel: a cohort study. Lancet Gastroenterol Hepatol 2017;2:23-31. 